\title{
Positron Annihilation Characteristics in Perfect and Imperfect Transition Metal Carbides and Nitrides
}

\author{
M.J. Puska, M. Šb*, G. Brauer** and T. Korhonen \\ Laboratory of Physics, Helsinki University of Technology, SF-02150 Espoo, Finland \\ * Institute of Physics of Materials, Academy of Sciences of the Czech Republic, Zižkova 22, CZ-616 62 \\ Brno, Czech Republic \\ ** Positron Annihilation Spectroscopy Group of the Technical University Dresden, Research Center \\ Rossendorf Inc., P.O. Box 510119, D-01314 Dresden, Germany
}

\begin{abstract}
First-principles electronic structure and positron state calculations for transition metal carbides and nitrides are performed. Perfect $\mathrm{NaCl}$-structures as well as structures with metal or carbon/nitrogen vacancies are considered. The positron affinities and lifetimes are determined. The trends are discussed and the results are compared with recent positron lifetime measurements for group IV and V refractory metal carbides and in hexagonal WC. The present analysis indicates, contradictory to an earlier interpretation, that positrons are trapped and annihilated both at carbon and metal vacancies. The concentration of metal vacancies seen by positrons is probably very low, below the sensitivity limit of the other experimental methods. Experimental data for WC may be well understood if a Bloch-like state in the lattice with carbon vacancies is employed as an initial positron state in the trapping model.
\end{abstract}

\section{INTRODUCTION}

Refractory metal carbides and nitrides exhibit interesting physical properties, e.g. high melting points, extreme hardness and relatively high superconducting transition temperatures. These make them attractive both for theoretical investigations and technological applications [1-4]. Many of their desirable characteristics are critically influenced by the presence of vacancies, which occur mostly on the non-metal sublattice. Experimental evidence shows that e.g. in the rock-salt (B1) modification of these compounds, up to $30 \%$ of the lattice sites may be vacant (i.e. up to $60 \%$ of the non-metal sublattice). Thus, the presence of vacancies in refractory metal carbides and nitrides seems to be an "intrinsic property" of these compounds $[3,4]$. In principle, vacancies may occur on both metal and non-metal sublattices.

Vacancy-free refractory metal carbides and nitrides would exhibit a very high electronic density. The corresponding positron lifetime may be estimated to be about $100 \mathrm{ps}$; this is lower than the values found for most transition metals. Recent positron lifetime measurements of Rempel et al. [5] yield the values of $155-176 \mathrm{ps}$ in refractory metal carbides, which were interpreted in terms of predominant positron annihilation with metal valence electrons, taking into account the presence of carbon vacancies even in nominally stoichiometric samples. It is one of the principal goals of this paper to find out, with the help of first-principles theoretical analysis, which types of vacancies are seen by positron in those materials, and to gain a deeper understanding of the experimental results. In addition to the refractory (group IV and V) metal carbides and nitrides, which exist in the B1 structure, we have extended our calculations along the 3d transition metal series up to $\mathrm{Fe}$ in order to see the trends exhibited by various quantities more clearly. It should be possible to generalize the main conclusions drawn for the B1-structure carbides and nitrides to the other structures as well. 
Another important quantity, which may be used in the analysis of the data obtained by positron annihilation spectroscopy (PAS), is the positron affinity. In a material with carbide or nitride precipitates, the difference in positron affinity between the precipitate and the matrix determines whether the precipitates are attractive or repulsive for the positron and, therefore, if they may be detected by PAS or not. In this connection, the present results are highly desired to understand the PAS data obtained in neutron-irradiated pressure vessel steels [6-8].

\section{COMPUTATIONAL METHOD}

Our self-consistent electronic structure calculations as well as the determination of the positron states are based on the density functional theory within the local density approximation (LDA) $[9,10]$. We calculate the self-consistent electronic structures of different carbides and nitrides using the linearmuffin-tin-orbital method (LMTO) within the atomic-spheres approximation (ASA). The details of the calculations may be found in [11]. In the calculations for the $\mathrm{NaCl}$-structure carbides and nitrides with vacancies a supercell with eight atomic sites (Fig. 1) is used. For the lattice constants the values optimized for the perfect crystals are employed. The atoms neighboring the vacancies are not allowed to relax from their perfect lattice positions. The positron states are calculated also in the supercell geometry using the same numerical methods. The position of the Fermi level defines the electron chemical potential $\mu_{-}$, whereas for positrons the position of the bottom $(a t k=0)$ of the lowest energy band relative to the crystal zero gives the positron chemical potential $\mu_{+}$. Both these quantities depend on the choice of the energy zero, which is, in our case, the ASA crystal zero level, defined as the zero of the Coulomb potential due to the nuclei and the electron density of the infinite solid [12].

The positron affinity $A_{+}$is obtained as the sum $A_{+}=\mu_{-}+\mu_{+}$. It does not depend on the choice of the energy zero. The positron lifetime $\tau$ is calculated from the electron and positron densities within LDA $[13-15]$ as

$$
\tau^{-1}=\lambda=\int d \mathbf{r} n_{+}(\mathbf{r}) \Gamma\left(n_{-}(\mathbf{r})\right),
$$

where $n_{+}(\mathbf{r})$ is the positron density and $\Gamma\left(n_{-}\right)$is the positron annihilation rate in a homogeneous electron gas with density $n_{-}$[10]. Let us emphasize that the present LDA calculations give firstprinciples results for both the positron affinities and the lifetimes, i.e. there are no adjustable parameters in our calculations.

\section{RESULTS AND DISCUSSION}

\subsection{Electronic structures and positron states}

The calculated electronic structures of transition metal carbides and nitrides both in the perfect state as well as with vacancies agree well with previous results whenever they exist; a more detailed discussion may be found in [11]. The values of the electron and positron chemical potentials calculated by the LMTO-ASA method are given in Table I. For the perfect lattices the positron chemical potential varies only slowly from one system to another. It decreases when the open volume seen by the positron increases. The introduction of metal vacancies lowers the positron chemical potential on the average by $3 \mathrm{eV}$, whereas the lowering by the smaller-sized carbon or nitrogen vacancies is only about $2 \mathrm{eV}$.

In order to visualize the positron states and to test the results for the positron lifetimes we have also applied the superimposed atom method [16] for the present systems. Here the electron density is non-self-consistent (it is given by the superposition of the free atom densities), but the benefit of this approach relative to the LMTO-ASA is that the real three-dimensional geometry is treated correctly (the potentials and charge densities are not spheridized). The positron densities in the perfect $\mathrm{TiC}$ lattice as well as in the lattices $\mathrm{Ti}_{3} \mathrm{C}_{4}$ and $\mathrm{Ti}_{4} \mathrm{C}_{3}$ containing $\mathrm{Ti}$ and $\mathrm{C}$ vacancies, respectively, are shown in Fig. 2. The plane of the figure is the (110) cut of the supercells used in the LMTO-ASA vacancy calculations (Fig. 1). In the perfect lattice the positron wave function has its maxima at the interstitial regions and vanishes towards the $\mathrm{Ti}$ or $\mathrm{C}$ nuclei. In case of the $\mathrm{Ti}$ vacancy the positron 


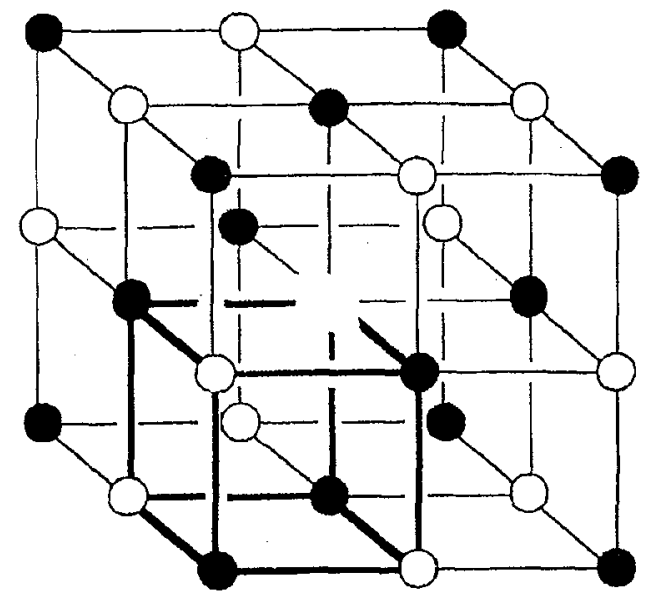

Fig. 1. $\mathrm{NaCl}$ structure with a vacancy. For a vacancy in the metal (non-metal) sublattice the white and black circles denote the metal (non-metal) and non-metal (metal) atoms, respectively. The supercell used in the calculations consists of eight sites forming a regular small cube. The sites are occupied by four black and three white circles and one site is vacant.

Fig. 2. Positron density in perfect $\mathrm{TiC}$ and the vacancy lattices $\mathrm{Ti}_{3} \mathrm{C}_{4}$ and $\mathrm{Ti}_{4} \mathrm{C}_{3}$. The panels show the regions of the (110) planes limited by the borders of the supercells used. For each panel the contour spacing is one sixth of the maximum value. Normalizing the positron densities so that there is one positron inside the supercell, the maximum positron densities are $0.0038 \mathrm{a}_{\mathrm{o}}^{-3}, 0.0139 \mathrm{a}_{\mathrm{o}}^{-3}$, and $0.0099 \mathrm{a}_{\mathrm{o}}^{-3}$ for $\mathrm{Ti}_{4} \mathrm{C}_{4}, \mathrm{Ti}_{3} \mathrm{C}_{4}$, and $\mathrm{Ti}_{4} \mathrm{C}_{3}$, respectively. The black triangles and squares denote the positions of the carbon and titanium nuclei, respectively.

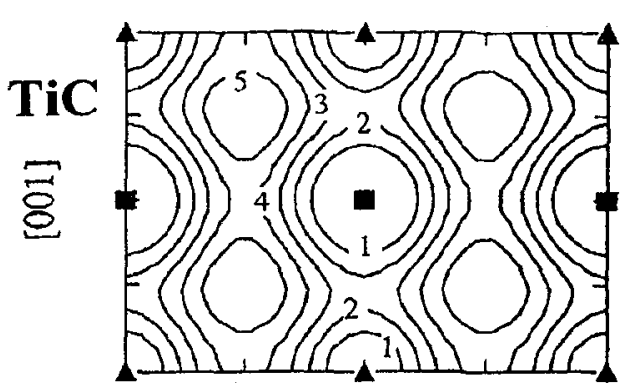

[110]

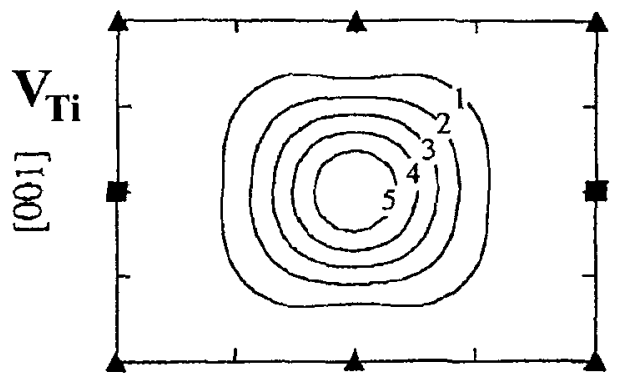

[110]

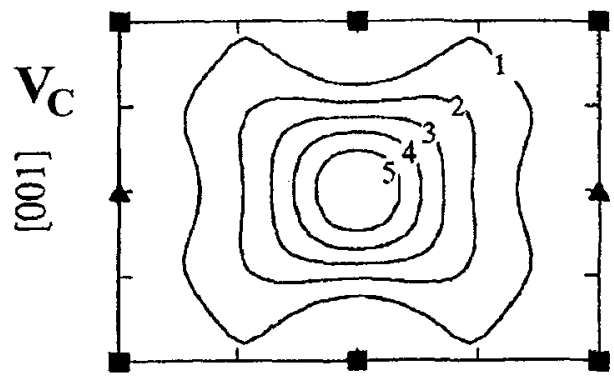

[110] 
Table I. Calculated properties of the $\mathrm{NaCl}$-structure transition metal carbides and nitrides. The perfect lattices ( $\mathrm{MeX}, \mathrm{Me}=$ transition metal, $\mathrm{X}=\mathrm{C}$ or $\mathrm{N}$ ) as well as lattices containing metal $\left(\mathrm{Me}_{3} \mathrm{X}_{4}\right)$ and carbon or nitrogen $\left(\mathrm{Me}_{4} \mathrm{X}_{3}\right)$ vacancies are considered. The results for the bcc $\mathrm{Fe}$ and orthorombic $\mathrm{Fe}_{3} \mathrm{C}$ with the experimental lattice constants are shown, too. For the carbides and nitrides the $\mathrm{NaCl}$-structure lattice constants $a$ are optimized for the perfect lattices. The experimental lattice constants [24] are given in the parentheses, for $\mathrm{Fe}_{3} \mathrm{C}$ the lattice parameters from [19] were used. $\mu_{-}$and $\mu_{+}$denote the electron and positron chemical potentials, respectively. $A_{+}$and $\tau$ are the positron affinity $\left(A_{+}=\mu_{-}+\mu_{+}\right)$and lifetime, respectively. The positron affinities and lifetimes differ slightly from the previously published values [6] due to a better self-consistency and more accurate determination of the positron potential in the present calculations.

\begin{tabular}{|c|c|c|c|c|c|}
\hline Material & $a\left(\mathrm{a}_{o}\right)$ & $\mu_{-}(\mathrm{eV})$ & $\mu_{+}(\mathrm{eV})$ & $A_{+}(\mathrm{eV})$ & $\tau(\mathrm{ps})$ \\
\hline TiC & $8.060(8.175)$ & +2.0 & $-\overline{-3.8}$ & -1.8 & $\overline{98}$ \\
\hline $\mathrm{Ti}_{3} \mathrm{C}_{4}$ & & -0.9 & -6.7 & -7.6 & 161 \\
\hline $\mathrm{Ti}_{4} \mathrm{C}_{3}$ & & +1.9 & -5.1 & -3.2 & 124 \\
\hline $\mathrm{VC}$ & $7.802(7.881)$ & +3.3 & -3.5 & -0.2 & 89 \\
\hline $\mathrm{V}_{3} \mathrm{C}_{4}$ & & +0.3 & -6.8 & -6.6 & 149 \\
\hline $\mathrm{V}_{4} \mathrm{C}_{3}$ & & +2.2 & -5.4 & -3.2 & 117 \\
\hline $\mathrm{CrC}$ & 7.641 & +3.1 & $-\overline{3.3}$ & -0.1 & 84 \\
\hline $\mathrm{Cr}_{3} \mathrm{C}_{4}$ & & +0.6 & -6.6 & -6.0 & 141 \\
\hline $\mathrm{Cr}_{4} \mathrm{C}_{3}$ & & +2.4 & -5.2 & -2.9 & 112 \\
\hline $\mathrm{MnC}$ & 7.544 & +2.8 & -3.2 & -0.4 & 82 \\
\hline $\mathrm{Mn}_{3} \mathrm{C}_{4}$ & & +0.8 & -6.5 & -5.7 & 136 \\
\hline $\mathrm{Mn}_{4} \mathrm{C}_{3}$ & & +2.2 & -5.1 & -2.9 & 109 \\
\hline $\mathrm{FeC}$ & 7.415 & +2.4 & -3.0 & -0.5 & 78 \\
\hline $\mathrm{Fe}_{3} \mathrm{C}_{4}$ & & +0.8 & -6.2 & -5.4 & 129 \\
\hline $\mathrm{Fe}_{4} \mathrm{C}_{3}$ & & +1.8 & -5.1 & -3.3 & 106 \\
\hline $\mathrm{ZrC}$ & $8.880(8.878)$ & +0.7 & -3.7 & -3.0 & 114 \\
\hline $\mathrm{Zr}_{3} \mathrm{C}_{4}$ & & -1.6 & -5.9 & -7.5 & 196 \\
\hline $\mathrm{Zr}_{4} \mathrm{C}_{3}$ & & +1.0 & -5.4 & -4.4 & 148 \\
\hline $\mathrm{NbC}$ & $8.620(8.447)$ & +1.9 & -2.3 & -0.4 & 105 \\
\hline $\mathrm{Nb}_{3} \mathrm{C}_{4}$ & & -0.2 & -4.8 & -5.0 & 181 \\
\hline $\mathrm{Nb}_{4} \mathrm{C}_{3}$ & & +1.2 & -4.8 & -3.5 & 138 \\
\hline $\mathrm{HfC}$ & $8.690(8.766)$ & +1.4 & -3.1 & -1.6 & 105 \\
\hline $\mathrm{Hf}_{3} \mathrm{C}_{4}$ & & -1.8 & -5.9 & -7.7 & 185 \\
\hline $\mathrm{Hf}_{4} \mathrm{C}_{3}$ & & +1.7 & -5.1 & -3.4 & 137 \\
\hline $\mathrm{TaC}$ & $8.566(8.420)$ & +3.0 & -2.9 & +0.1 & 99 \\
\hline $\mathrm{Ta}_{3} \mathrm{C}_{4}$ & & -0.5 & -6.3 & -6.8 & 177 \\
\hline $\mathrm{Ta}_{4} \mathrm{C}_{3}$ & & +1.8 & -5.6 & -3.8 & 130 \\
\hline TiN & $8.143(8.013)$ & +5.0 & $-\overline{3.8}$ & +1.2 & 100 \\
\hline $\mathrm{Ti}_{3} \mathrm{~N}_{4}$ & & +0.5 & -7.4 & -6.9 & 159 \\
\hline $\mathrm{Ti}_{4} \mathrm{~N}_{3}$ & & +3.5 & -6.0 & -2.6 & 143 \\
\hline$V N$ & $7.968(7.826)$ & +4.5 & -3.5 & +1.0 & 94 \\
\hline $\mathrm{V}_{3} \mathrm{~N}_{4}$ & & +0.8 & -7.1 & -6.2 & 154 \\
\hline $\mathrm{V}_{4} \mathrm{~N}_{3}$ & & +3.4 & -5.6 & -2.2 & 138 \\
\hline $\mathrm{CrN}$ & 7.830 & +3.8 & -3.5 & +0.3 & 89 \\
\hline $\mathrm{Cr}_{3} \mathrm{~N}_{4}$ & & +0.6 & -7.0 & -6.5 & 147 \\
\hline $\mathrm{Cr}_{4} \mathrm{~N}_{3}$ & & +3.4 & -5.5 & -2.1 & 123 \\
\hline $\mathrm{Fe}$ & 5.406 & -1.3 & -3.1 & -4.4 & 99 \\
\hline $\mathrm{Fe}_{3} \mathrm{C}$ & & +1.9 & -2.5 & -0.6 & 91 \\
\hline
\end{tabular}


density is clearly localized around the vacant site, whereas for the $\mathrm{C}$ vacancy positron exhibits a stronger diffusion towards the supercell boundaries. This means that the effects due to the finite supercell size are expected to be stronger for the carbon or nitrogen vacancies than for the metal vacancies. However, the localization of the positron density in the vacancies seen in Fig. 2 means that the positron results, for example the lifetimes obtained, may be considered also as results for isolated vacancies.

\subsection{Positron lifetimes and affinities}

The calculated positron lifetimes for the different transition metal carbides and nitrides are given in Table I, for both undefected and defected lattices. The positron lifetimes in perfect transition metal carbides and nitrides are low, substantially lower than the calculated [13-15] or experimental [17] lifetimes in the corresponding pure metals. These low values reflect their high atomic and electronic densities. There is a charge transfer of about one electron from a metal to a non-metal sphere. Because of this and the smaller ionic core size of non-metal the positron resides more in the nonmetal spheres and, therefore, the annihilation takes place preferably in the non-metal spheres than in the metal spheres. Due to the charge transfer, metal vacancies may be considered as more negative environments (they are surrounded by negative non-metal spheres) whereas non-metal vacancies are more positive. A more detailed analysis of the charge distribution is performed in [11].

The calculated positron affinities are given in Table $\mathrm{I}$ as well. Their importance consists in the fact that the difference of the positron energies between different materials in contact is equal to the difference in their positron affinities [12]. For the perfect lattices the affinities are higher than those for the most elemental metals [12]. This reflects again high electronic densities in carbides and nitrides. For the materials with carbon or nitrogen vacancies the positron affinities are about $3 \mathrm{eV}$ lower than the affinities for perfect crystals. The decrease of the positron affinity due to metal vacancies is stronger, on the average about $6 \mathrm{eV}$. The high positron affinities for the perfect carbide and nitride lattices suggest that these materials could be used as effective moderators in the slow positron beam technique. The problem may, however, be the difficulty to produce high-quality single crystals with a low vacancy concentration.

In Table I we show data for the bcc Fe, too. This is because the transition metal carbides are thought to be the irradiation-induced precipitates in reactor pressure vessel steels. Precipitates formed by neutron irradiation have already been studied by PAS [18]. According to Table I the carbicle precipitates in Fe could trap positrons and be observable only if they contain metal vacancies because only in that case their affinity to the positron is lower (more negative) than the iron affinity. In order to approach closer the reality in steels we have also performed calculations for the compound $\mathrm{Fe}_{3} \mathrm{C}$, cementite [11]. The corresponding positron annihilation results are shown at the bottom of Table I. It is seen that the Fermi level in $\mathrm{Fe}_{3} \mathrm{C}$ is quite high relative to bcc Fe. This fact, in addition to the increase of the positron chemical potential, decreases the absolute value of the positron affinity and makes the positron trapping at perfect $\mathrm{Fe}_{3} \mathrm{C}$ precipitates in steels impossible. Due to a large computation cost, we have not performed calculations for vacancies in $\mathrm{Fe}_{3} \mathrm{C}$, but we expect that their properties, with respect to positron states, will be qualitatively similar to those for FeC. The consequences of the present positron affinity data in the interpretation of the PAS results for irradiated reactor pressure vessel steels are discussed in another context [6-8].

\subsection{Comparison with positron lifetime measurements}

Let us compare the present calculated positron lifetimes with recent measurements of Rempel et al. [5] for group IV and group V transition metal carbides in Table II. The theoretical and experimental positron lifetimes for the corresponding transition metals are shown for a comparison, too. It may be seen that the experimental lifetimes are much longer than the lifetimes calculated here for perfect carbide lattices.

A more detailed comparison of the theoretical and experimental results shows that, with a surprisingly good accuracy, the measured lifetimes correspond to the average of the lifetimes calculated in the 
Table II. Comparison of the theoretical and experimental positron lifetimes for the $\mathrm{NaCl}$ structure transition metal carbides. The configurations $\mathrm{Me}_{3} \mathrm{C}_{4}$ and $\mathrm{Me}_{4} \mathrm{C}_{3}$ correspond to metal and carbon vacancies, respectively. The lifetimes calculated [15] for the transition metals are shown for comparison. $\tau_{t h e o}$ is the calculated lifetime and $\tau_{\text {exp }}$ denotes the experimental lifetime. For a given carbide, $\tau_{v}$ is the mean value of the theoretical positron lifetimes corresponding to the metal and carbon vacancies.

\begin{tabular}{|c|c|c|c|}
\hline Material & $\tau_{\text {theo }}(\mathrm{ps})$ & $\tau_{v}(\mathrm{ps})$ & $\tau_{\exp }(\mathrm{ps})$ \\
\hline$\overline{\mathrm{TiC}}$ & $\overline{98}$ & 143 & $160^{a}(\mathrm{TiC})$ \\
\hline $\mathrm{Ti}_{3} \mathrm{C}_{4}$ & 161 & & \\
\hline $\mathrm{Ti}_{4} \mathrm{C}_{3}$ & 124 & & \\
\hline $\mathrm{Ti}$ & $146^{b}$ & & $147^{a}$ \\
\hline $\mathrm{ZrC}$ & 114 & 172 & $176^{a}\left(\mathrm{ZrC}_{0.98}\right)$ \\
\hline $\mathrm{Zr}_{3} \mathrm{C}_{4}$ & 196 & & \\
\hline $\mathrm{Zr}_{4} \mathrm{C}_{3}$ & 148 & & \\
\hline $\mathrm{Zr}$ & $159^{b}$ & & $165^{a}$ \\
\hline $\mathrm{NbC}$ & 105 & 159 & $161^{a}(\mathrm{NbC})$ \\
\hline $\mathrm{Nb}_{3} \mathrm{C}_{4}$ & 181 & & $151^{a}\left(\mathrm{NbC}_{0.72}\right)$ \\
\hline $\mathrm{Nb}_{4} \mathrm{C}_{3}$ & 138 & & \\
\hline $\mathrm{Nb}$ & $122^{6}$ & & $119^{a}$ \\
\hline $\mathrm{HfC}$ & 105 & 161 & $173^{a}(\mathrm{HfC})$ \\
\hline $\mathrm{Hf}_{3} \mathrm{C}_{4}$ & 185 & & \\
\hline $\mathrm{Hf}_{4} \mathrm{C}_{3}$ & 137 & & \\
\hline $\mathrm{Hf}$ & $149^{6}$ & & $174^{a}$ \\
\hline $\mathrm{TaC}$ & 99 & 154 & $155^{a}\left(\mathrm{TaC}_{0.99}\right)$ \\
\hline $\mathrm{Ta}_{3} \mathrm{C}_{4}$ & 177 & & \\
\hline $\mathrm{Ta}_{4} \mathrm{C}_{3}$ & 130 & & \\
\hline $\mathrm{Ta}$ & $117^{6}$ & & $116^{a}$ \\
\hline $\mathrm{WC}^{d}$ & 95 & & $118,158^{c}$ \\
\hline $\mathrm{W}_{3} \mathrm{C}_{4}$ & 161 & & \\
\hline $\mathrm{W}_{4} \mathrm{C}_{3}$ & 116 & & \\
\hline $\mathrm{W}_{31} \mathrm{C}_{32}$ & 176 & & \\
\hline $\mathrm{W}_{32} \mathrm{C}_{31}$ & 118 & & \\
\hline W & & & $105^{e}$ \\
\hline
\end{tabular}

${ }^{a}$ Ref.[5] ${ }^{b}$ Ref.[15] $\quad{ }^{c}$ Ref. [23], see also discussion in the text. ${ }^{d}$ Calculations were performed for the $\mathrm{NaCl}$ structure. $\quad{ }^{e}$ Ref. [17]

structures with metal and carbon vacancies ( $\tau_{v}$ in Table II). Only in case of TiC the measured lifetime seems to correspond well to the lifetime for a titanium vacancy. On the basis of these results, it seems that in $\mathrm{TiC}$ positrons annihilate mostly at the $\mathrm{Ti}$ vacancies, whereas in the other carbides the annihilation takes place both at metal and carbon vacancies.

There is also another argument in favour for positron annihilation at vacancies and not in the bulk. In 1980, Manuel [20] measured two-dimensional angular correlation positron annihilation spectra of $\mathrm{NbC}$, which were fully isotropic. He interpreted his results as the indication of positron trapping at vacancies, as no modulation due to bulk electronic momentum density was present [21].

Thus, the comparison of our theoretical positron lifetimes with the measured ones strongly indicates the existence of metal vacancies in the samples of group IV and group V refractory metal carbides. Their concentration may, however, be small in comparison with that of the carbon vacancies as, according to the affinity results, the positron has a much larger preference for annihilation at the metal vacancies than at the carbon vacancies.

An independent (i.e. a non-positron) evidence for the existence of metal vacancies in B1-type refrac- 
tory metal carbides and nitrides is rare. In refractory metal nitrides the existence of metal vacancies has been independently proved, in carbides they may exist as well according to those measurements, but in a small amount (see discussion in [11] and the references therein). From comparison of our calculations with the experimental results [5] we are not able to estimate the metal vacancy concentration in refractory metal carbides either. It will be probably lower than 0.005 , the estimated sensitivity limit of the pycnometric and X-ray diffraction methods [22]. As the samples contained about 1 at. $\%$ of oxygen [22] and the concentration of metal vacancies in refractory metal oxides can be up to several percent, it is not excluded that the existence of metal vacancies we predict in carbides of the group IV and group V refractory metals is connected with the presence of oxygen impurities.

In a very recent paper [23], Rempel et. al. reported results of the lifetime measurements in hexagonal WC. On the basis of one-defect trapping model, they determined the bulk positron lifetime to be $118 \pm 3 \mathrm{ps}$, the defect component being $158 \pm 3$ ps and the shorter component of about 90 ps [22].

As a preliminary theoretical estimate, we performed the superimposed atom method calculation for $\mathrm{WC}$ in $\mathrm{NaCl}$ structure with the same lattice volume as in the hexagonal structure. One can suppose that the lifetimes in the hexagonal structure will not be too much different from those in the $\mathrm{NaCl}$ structure.

The results are collected at the bottom of Table II. The calculated bulk lifetime of 95 ps equals to the estimate of Ref. [23] and is very similar to the values for $\mathrm{TiC}$ and $\mathrm{TaC}$. The value of $118 \pm 3 \mathrm{ps}$ interpreted in [23] as the experimental bulk positron lifetime coincides surprisingly well with our value for annihilation at carbon vacancies, regardless of the size of the supercell. The defect component of $158 \pm 3$ ps corresponds satisfactorily to the calculated metal vacancy lifetime.

This comparison suggests a conclusion that in WC the initial positron state for the trapping model might be a Bloch-like (quasiperiodic) state in the lattice with carbon vacancies, having thus an appreciable amount of localization at those vacancies. This idea is supported by relatively high values of the amplitude of positron wave function at the supercell boundary: for the $\mathrm{W}_{4} \mathrm{C}_{3}$ and $\mathrm{W}_{32} \mathrm{C}_{31}$ supercells, the maxima of the positron wave function at the cell boundaries amount to about one third and one tenth of its value at the center of vacancy, respectively. In this model the lifetime of 118 ps for a carbon vacancy system would serve as the bulk positron lifetime in a relevant one-defect trapping model, where the "true" defects (in the sense of the trapping model) would be something else than carbon vacancies, e.g. metal vacancies, as it is probably the case in WC. A similar situation could take place in all those solids where the defect-free bulk cannot be prepared anyhow, as e.g. in refractory metal carbides. The validity of this assumption must be, however, verified in the future.

\section{CONCLUSIONS}

We have performed first-principles electronic structure and positron state calculations for transition metal carbides and nitrides. The quantities studied are the positron affinity and lifetime. For the perfect carbide or nitride lattices the positron affinities are high and the lifetimes are short in comparison with the values for corresponding transition metals. This result reflects a very high atomic and electronic density of the transition metal carbides and nitrides. For the lattices with metal or carbon/nitrogen vacancies the positron affinities are lower and the positron lifetimes longer than for the perfect lattices. The changes are larger for the lattices with metal vacancies.

The comparison of the calculated positron lifetimes with the recent measurements for group IV and group V refractory metal carbides [5] and for WC [23] shows that even in the highly stoichiometric systems positrons annihilate mainly at vacancies. Our results indicate that positrons are trapped and annihilated also at metal vacancies, the existence of which is difficult to see by other experimental methods. The results for WC suggest a new view on the initial positron state in trapping model - it could be a Bloch-like state in the lattice with the carbon vacancies.

The positron affinities calculated in this work are important parameters if used in the interpretation of the positron annihilation results for materials containing carbide or nitride precipitates. A discussion 
about irradiation-induced precipitates in reactor pressure vessel steels, which might consist of carbides and/or nitrides, is published elsewhere [6-8].

\section{Acknowledgements}

The authors wish to thank Dr. A. A. Rempel for stimulating discussions and for communicating his results prior to publication, and Dr. A. Manuel for information about his two-dimensional angular correlation positron annihilation measurements on NbC.

\section{References}

[1] L. E. Toth, Transition Metal Carbides and Nitrides (Academic Press, New York-London, 1971).

[2] K. Schwarz, CRC Critical Rev. in Sol. State and Mat. Sci. 13, 211 (1987).

[3] A. I. Gusev, phys. stat. sol (b) 163, 17 (1991); A. I. Gusev and A. A. Rempel, phys. stat. sol. (a) 135,15 (1993).

[4] A. A. Rempel, Ordering Effects in Non-Stoichiometric Interstitial Compounds (Nauka, Ural Division, Ekaterinburg, 1992) (in Russian).

[5] A. A. Rempel, M. Forster and H.-E. Schaefer, J. Phys.: Condens. Matter 5, 261 (1993); for a more detailed presentation see A. A. Rempel, M. Forster and H.-E. Schaefer, Dokl. Akad. Nauk SSSR 326, 91 (1992).

[6] G. Brauer, M. Šb and M. Puska, Mat. Sci. Forum 105-110,611 (1992). This paper is a preliminary report of the results presented in [7]. The values of positron affinities and lifetimes differ slightly from those in [7] because of a better self-consistency and more accurate positron potential in the more recent calculations [7].

[7] G. Brauer, M. J. Puska, T. Korhonen, and M. Šob, submitted to Nucl. Enginrg. Design.

[8] G. Brauer, this Proceedings.

[9] For a recent review, see R. O. Jones and O. Gunnarsson, Rev. Mod. Phys, 61, 689 (1989).

[10] E. Boroński and R. M. Nieminen, Phys. Rev. B 34, 3820 (1986).

[11] M. J. Puska, M. Šob, G. Brauer and T. Korhonen, Phys Rev. B 49, 10947 (1994).

[12] M. J. Puska, P. Lanki, and R. M. Nieminen, J. Phys.: Condens. Matter 1, 6081 (1989).

[13] K. O. Jensen, J. Phys.: Condens. Matter 1, 10595 (1989).

[14] S. Daniuk, M. Šb, and A. Rubaszek, Phys. Rev, B 43, 2580 (1991).

[15] M. J. Puska, J. Phys.: Condens. Matter 3, 3455 (1991).

[16] M. J. Puska and R. M. Nieminen, J. Phys. F: Metal Phys. 13, 333 (1983).

[17] A. Seeger, F. Banhart and W. Bauer, in Positron Annihilation, eds. L. Dorikens-Vanpraet, M. Dorikens and D. Segers (World Scientific, Singapore, 1989), p. 275; A. Seeger and F. Banhart, phys. stat. sol. (a) 102, 171 (1987).

[18] G. Brauer, L. Liszkay, B. Molnar and R. Krause, Nucl. Enginrg. Design 127, 47 (1991).

[19] R. W. G. Wyckoff, Crystal Structures, Vol. 2: Inorganic Compounds $\mathrm{RX}_{n}, \mathrm{R}_{n} \mathrm{MX}_{2}, \mathrm{R}_{n} \mathrm{MX}_{3}$ (John Wiley and Sons, New York, 1964).

[20] A. Manuel, private communication.

[21] A. Alam, J. H. Kaiser, P. A. Walters, R. L. Waspe and R. N. West, in Positron Annihilation, eds. P. G. Coleman, S. C. Sharma and L. M. Diana (North Holland, Amsterdam, 1982), p. 331.

[22] A. A. Rempel, private communication.

[23] A. A. Rempel, H.-E. Schaefer, M. Forster and A. I. Girka, in Covalent Ceramics II: NonOxides, eds. A. R. Barron, G. S. Fischman, M. A. Fury and A. F. Hepp, Mat. Res. Soc. Symp. Proc. vol. 326, Materials Research Society, Pittsburgh 1994, to be published.

[24] The experimental lattice constants for carbides are those given in Ref. [5], for VC in Ref. [25], for TiN in A. N. Christiansen, Acta Chem. Scand. A 32, 87 (1978) and for VN in B. R. Zhao, L. Chen, and H. L. Luc, Phys. Rev. B 29, 6198 (1984).

[25] V. P. Zhukov, V. A. Gubanov, O. Jepsen, N. E. Christensen, and O. K. Andersen, J. Phys. Chem. Solids, 49, 841 (1988). 